\title{
Growth and Characterization of High-Quality GaN Nanowires on PZnO and PGaN by Thermal Evaporation
}

\author{
L. Shekari, ${ }^{1}$ H. Abu Hassan, ${ }^{1}$ S. M. Thahab, ${ }^{2}$ and Z. Hassan ${ }^{1}$ \\ ${ }^{1}$ Nano-Optoelectronics Research and Technology Laboratory, School of Physics, Universiti Sains Malaysia, \\ 11800 USM, Penang, Malaysia \\ ${ }^{2}$ Materials Engineering Department, College of Engineering, University of Kufa, 00964-21 Najaf, Iraq
}

Correspondence should be addressed to L. Shekari, 1sg09_phy089@student.usm.my

Received 10 June 2011; Revised 13 August 2011; Accepted 27 August 2011

Academic Editor: Kui-Qing Peng

Copyright ( $) 2011$ L. Shekari et al. This is an open access article distributed under the Creative Commons Attribution License, which permits unrestricted use, distribution, and reproduction in any medium, provided the original work is properly cited.

\begin{abstract}
In the current research, an easy and inexpensive method is used to synthesize highly crystalline gallium nitride (GaN) nanowires (NWs) on two different substrates [i.e., porous zinc oxide (PZnO) and porous gallium nitride (PGaN)] on Si (111) wafer by thermal evaporation without any catalyst. Microstructural studies by scanning electron microscopy and transmission electron microscope measurements reveal the role of the substrates in the nucleation and alignment of the GaN NWs. Further structural and optical characterizations were performed using high-resolution X-ray diffraction, energy-dispersive X-ray spectroscopy, and photoluminescence spectroscopy. Results indicate that the NWs have a single-crystal hexagonal GaN structure and growth direction in the (0001) plane. The quality and density of GaN NWs grown on different substrates are highly dependent on the lattice mismatch between the NWs and their substrates. Results indicate that NWs grown on PGaN have better quality and higher density compared to NWs on PZnO.
\end{abstract}

\section{Introduction}

Group III nitrides have emerged in the past decade as materials of choice for blue and UV optoelectronic devices, as well as high-power, high-frequency electronics. Knowledge of the surface electronic structure and its dependence on the surface polarity and growth conditions is of fundamental importance [1]. One-dimensional growth of wide bandgap semiconductor such as $\mathrm{GaN}(3.4 \mathrm{eV})$ nanostructures holds considerable technological applications for nanodevices, such as LEDs and solar cells [2-4].

Several methods to grow one-dimensional GaN nanostructures have been developed to account for the catalystinduced gallium nitride $(\mathrm{GaN})$ nanowires (NWs). Nevertheless, recent papers have found that NW contamination caused by the catalyst considerably limits the structural and optical quality of NWs. However, only a small number of basic works have been devoted to the investigation of the physical mechanisms that account for the self-induced growth of GaN NWs [5].

Recently vapour phase and chemical methods dominate NW growth to produce semiconductor devices [6-9]. These methods are steady-state techniques and provide a better control of NWs' growth, in contrast to solution methods [10]. But these methods are expensive and also are not easy to do. So, the most important focus on current photovoltaic cell research is finding new methods to improve device efficiency, safety, and cost.

Herein we report an easy, cheap, and successful method, based on thermal evaporation method in an atmosphere of argon (Ar) gas (no ammonia gas was used), to produce highly crystalline GaN NWs on PZnO and PGaN substrates. The influences of the substrates, $\mathrm{PZnO}$ and PGaN, on the composition and morphology of these NWs have been investigated.

\section{Experiment}

Alumina boats were used to hold the commercial GaN powder and the substrates. Thermal evaporation was used to produce GaN NWs under the flow of argon (Ar) gas, without any catalyst to assist in the preparation. The NWs were synthesized inside a tube furnace for $2 \mathrm{~h}$. In each experiment, 


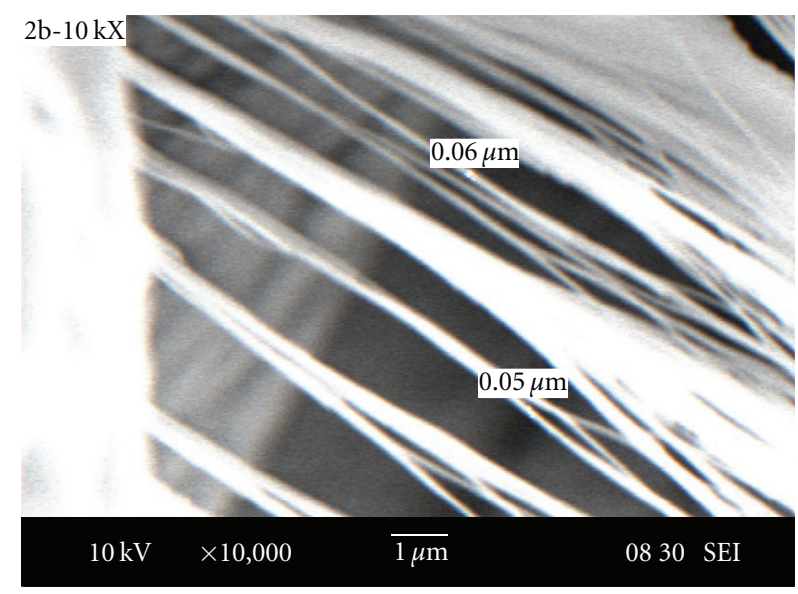

(a)

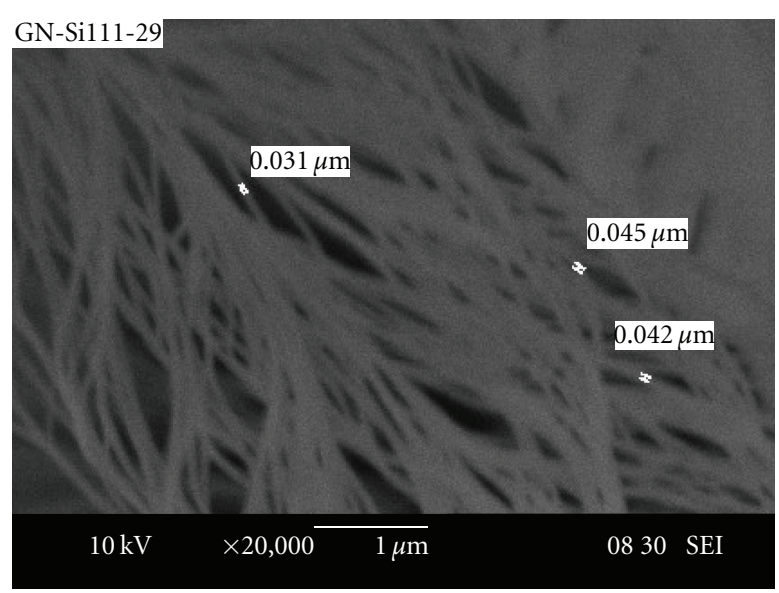

(b)

FIGURe 1: SEM images of GaN NWs on (a) PZnO substrate and (b) PGaN substrate.

the substrates were placed in the second boat at a distance of $6 \mathrm{~cm}$ from the powder, which was placed in the center of the first boat. Two sets of experiments were conducted. In the first set, porous zinc oxide ( $\mathrm{PZnO}$ ) was used as substrate, and in the second set, porous gallium nitride $(\mathrm{PGaN})$ was used. The size of each substrate was approximately $1 \times 1 \mathrm{~cm}^{2}$; one substrate was used in each experiment. The Ar flow was $1.5 \mathrm{sccm}$.

The substrates were first washed and cleaned using the standard RCA cleaning procedure. Next, the substrates were ultrasonically cleaned with acetone and isopropyl alcohol (IPA), rinsed with distilled water, and dried using a nitrogen gas blow system. The temperature of the heating region where the substrates were placed was $1150^{\circ} \mathrm{C}$. A flow of $\mathrm{Ar}$ gas was initially used to purify the tube for $15 \mathrm{~min}$ before placing the samples into the furnace and introducing back the gas flow into the system. The furnace was turned off to allow it to cool to room temperature. The samples were removed from the furnace after $20 \mathrm{~min}$.

The structures of the as-grown samples were then characterized by a scanning electron microscope (SEM, JEOL JSM-6460LV) operated at $10 \mathrm{kV}$ and by transmission electron microscope (TEM, FEI CM 12) operated at $120 \mathrm{kV}$. Further structural and optical characterizations were performed using high-resolution X-ray diffraction (HR-XRD), energydispersive X-ray spectroscopy (EDX), and room temperature photoluminescence spectroscopy (PL) with He-Cd laser $(\lambda=325 \mathrm{~nm})$ using a HORIBA JOBIN YVON-HR800 UV microspectrophotometer with a resolution of $1 \mathrm{~cm}^{-1}$.

\section{Results and Discussion}

The SEM images in Figures $1(\mathrm{a})$ and 1(b) illustrate the growth distribution of the NWs on the PZnO and PGaN substrates, respectively. The diameters of the wires were approximately $50-60 \mathrm{~nm}$ for the samples on $\mathrm{PZnO}$, whereas the diameters for GaN NWs on PGaN (approximately 30$40 \mathrm{~nm}$ ) were less than those on the PZnO.
For TEM observation, the GaN NWs were pulverized in ethanol $100 \%$ and then dispersed onto a copper grid. In Figures 2(a) and 2(b), the TEM images of GaN NWs clearly confirm that the average diameters of the NWs on PGaN were less than those on the PZnO.

TEM images reveal that the geometrical shape of the $\mathrm{GaN}$ nanostructures is similar to a wire. Also these images illustrate uniform diameter sizes. Careful TEM observation indicates that all NWs are composed of only one layer of sheet growing along the wire axis, as shown in Figure 2. Moreover, this figure indicates that there is no difference in thickness between the center and edges.

Perfect one-dimensional growth is preferable for using in most device applications, such as solar cells. For example, twinning, stacking faults, and high-order grain boundaries are frequently observed in GaN NWs, and these highdensity defects are believed to play an important role in the unidirectional formation and growth of the GaN NWs $[11,12]$. Also the growth direction of the NWs is important because the orientation of the NWs is related to their properties [11].

EDX microanalysis of the samples grown on $\mathrm{PZnO}$, shows $\mathrm{Zn}, \mathrm{O}, \mathrm{Ga}, \mathrm{N}$, and $\mathrm{Si}$ atoms (Figure 3(a)). However, Figure 3(b) shows EDX analysis of the NWs grown on PGaN, which indicates the presence of $\mathrm{Ga}, \mathrm{N}, \mathrm{Si}$, and $\mathrm{O}$ atoms among which $\mathrm{Si}$ and $\mathrm{O}$ are from the $\mathrm{Si}$ substrates and the oxide layer. For the PGaN, silicon molecules diffused into the $\mathrm{GaN}$ porous structure and replaced the position of $\mathrm{Ga}$, which decreased the crystallization temperature of $\mathrm{GaN}$ [13]. At high temperatures $\left(T>900^{\circ} \mathrm{C}\right)$, Si tends to remain at $\mathrm{Ga}$ sites and acts as donor [14]. A thin layer of silicon oxide was detected in the interface between GaN and the Si wafer. The diffusion of oxygen during deposition into Si wafer caused the formation of silicon oxide. No other impurities were detected. For the two samples grown on both $\mathrm{PZnO}$ and PGaN, wurtzite GaN has a higher Si content and a lower Ga and $\mathrm{N}$ content.

Figure 4 shows typical room-temperature PL spectra of the GaN NWs with He-Cd laser used as an excitation source 


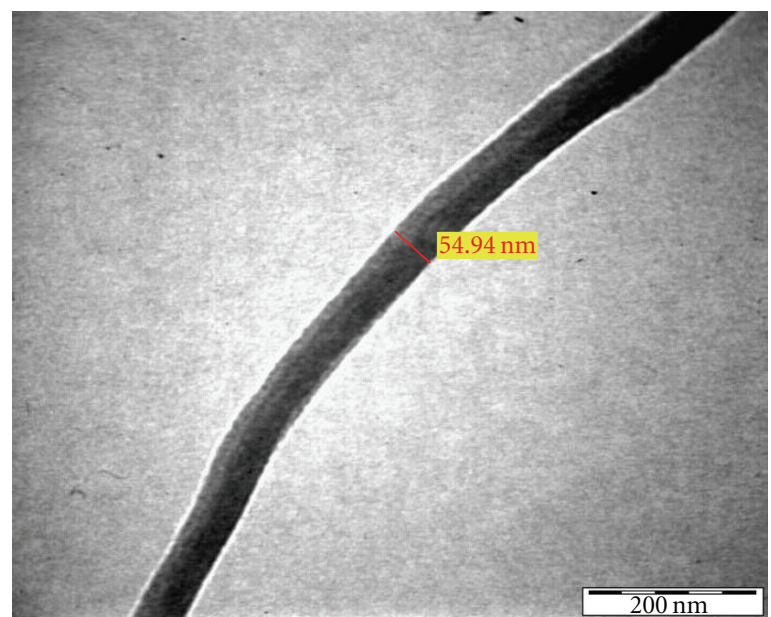

(a)

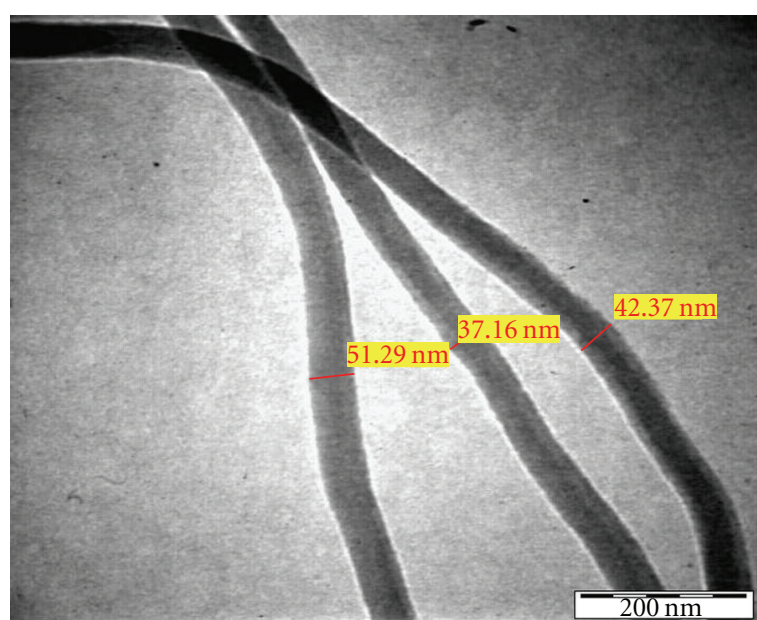

(b)

Figure 2: TEM images of GaN NWs on (a) PZnO substrate and (b) PGaN substrate.

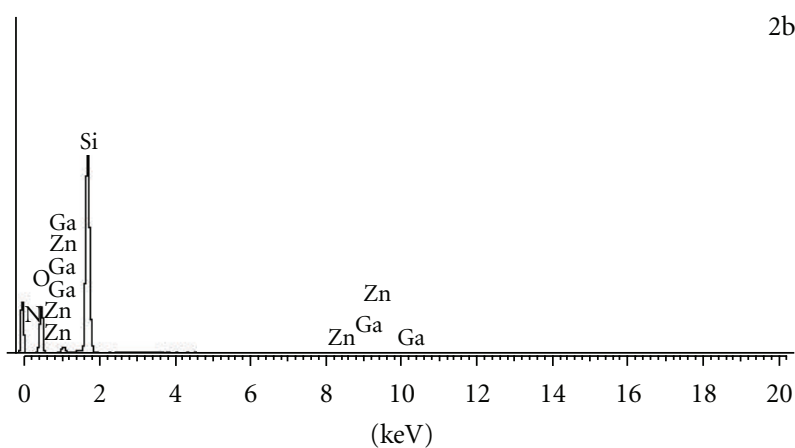

(a)

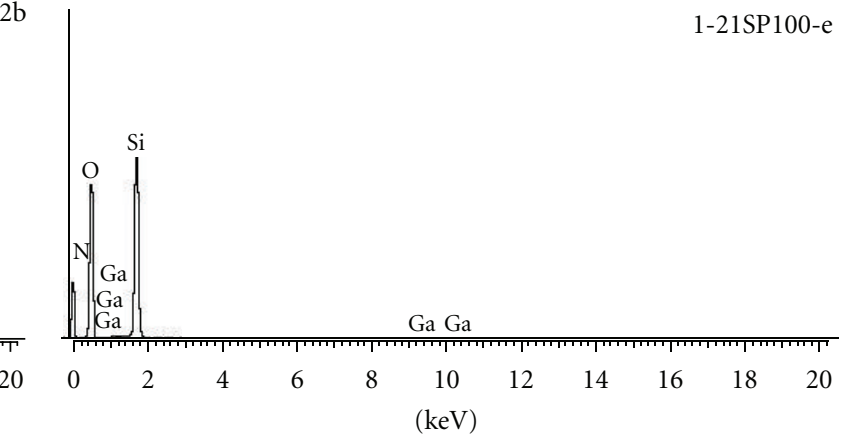

(b)

Figure 3: EDX spectra of GaN NWs on (a) PZnO substrate and (b) PGaN substrate.

(325 nm wavelength). Figure 4(a) shows a dominant broad peak of as-grown PZnO located at $369.8 \mathrm{~nm}$, and Figure 4(b) shows the PL peak of GaN NWs on PZnO at $368.4 \mathrm{~nm}$. These peaks represent band-to-band recombination in $\mathrm{GaN}$ NWs. The as-grown PZnO shows prominent green emission lines. The green emission lines were essentially caused by the defects that resulted during $\mathrm{ZnO}$ porous fabrication [15].

Figures 4(c) and 4(d), respectively, show that the dominant broad peak of as-grown PGaN is located at $352.9 \mathrm{~nm}$ and that of the GaN NWs on PGaN is at $362.8 \mathrm{~nm}$. No other peaks were found in the PL spectra, indicating that the assynthesized GaN nanostructures have good optical quality and few defects. Focusing on the PL of the GaN NWs shows that, on the surfaces of the $\mathrm{PZnO}$ and $\mathrm{PGaN}$, there are fibrous NW structures that can increase the number of luminescent centers which can increase the PL of the GaN NWs due to additional electron-hole pairs formed for recombination.

The broadening of the energy bandgaps at $368.4 \mathrm{~nm}$ $(3.37 \mathrm{eV})$ and $362.8 \mathrm{~nm}(3.42 \mathrm{eV})$ for $\mathrm{GaN} \mathrm{NWs}$ on $\mathrm{PZnO}$ and PGaN, respectively, is due to quantum confinement effects of the NWs crystalline structure and different degree of quantum confinement in different GaN NWs that affects the electron-hole pair recombination [16].

The GaN NWs show a very good emission property, which is a great advantage for laser device applications. Comparing the PL of GaN nanostructure on both porous substrates to the PL spectrum of the as-grown substrate shows that the intensities of the GaN NWs peaks are significantly less than the intensity of the PL peaks of their as-grown substrates. The decrease in intensity of these peaks during annealing to produce GaN NWs is due to diminishing atomic ordering with heating.

Figure 5 shows the HR-XRD spectra of the as-grown $\mathrm{PZnO}, \mathrm{PGaN}$, and GaN NWs grown on PZnO and PGaN. The rather sharp peaks can be indexed to the hexagonal wurtzite structure of GaN NWs with lattice constants $a / b=$ $3.1350 \AA$ and $c=5.1190 \AA$. These values are close to the reported values of bulk GaN crystals [17].

Figures 5(a) and 5(b) show the X-ray diffraction patterns of the as-grown $\mathrm{PZnO}$ and $\mathrm{PGaN}$ wafers, respectively. These figures confirm the formation of hexagonal wurtzite porous $\mathrm{ZnO}$ and Porous GaN. Figure 5(a) shows two peaks attributed to PZnO: higher intensity (0002) and lower 


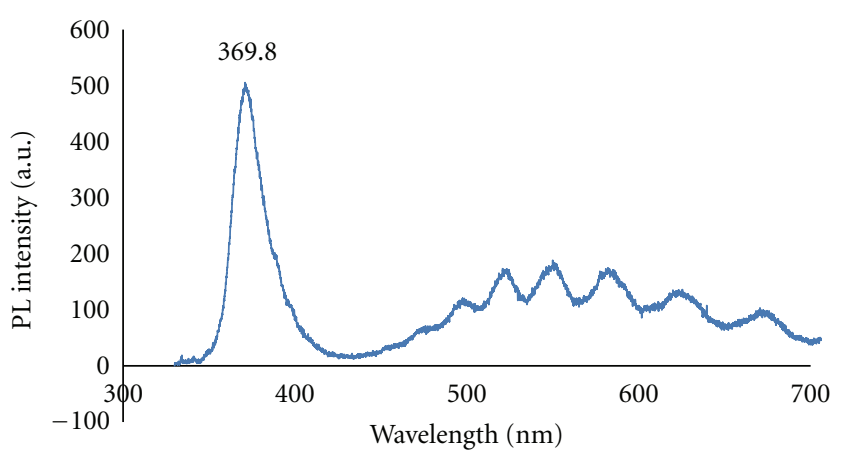

(a)

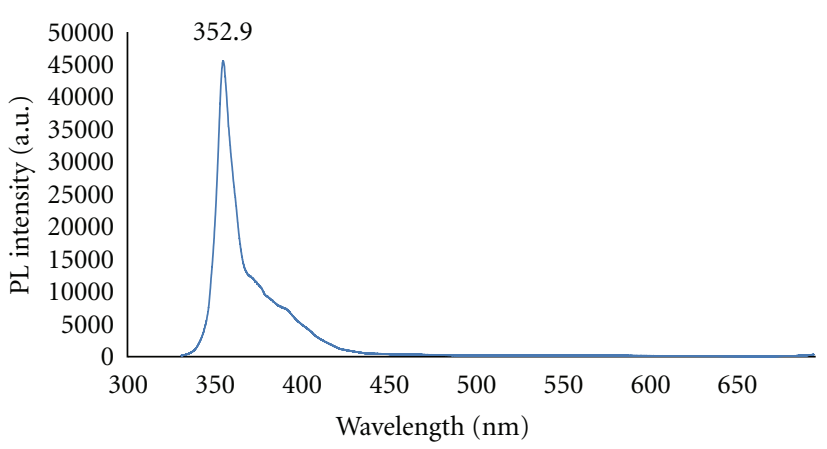

(c)

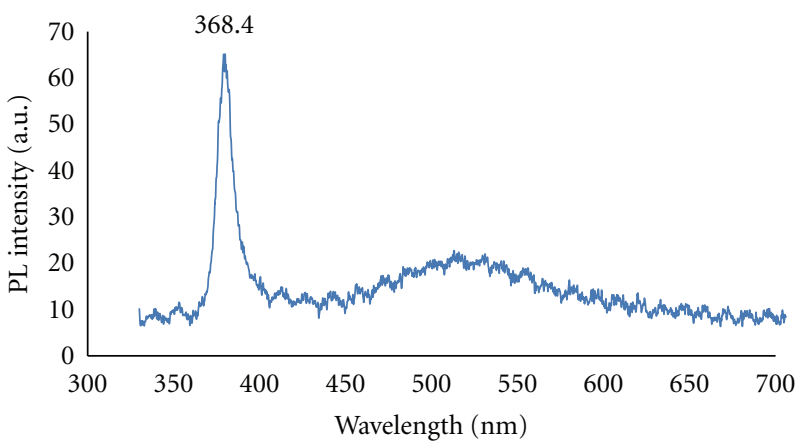

(b)

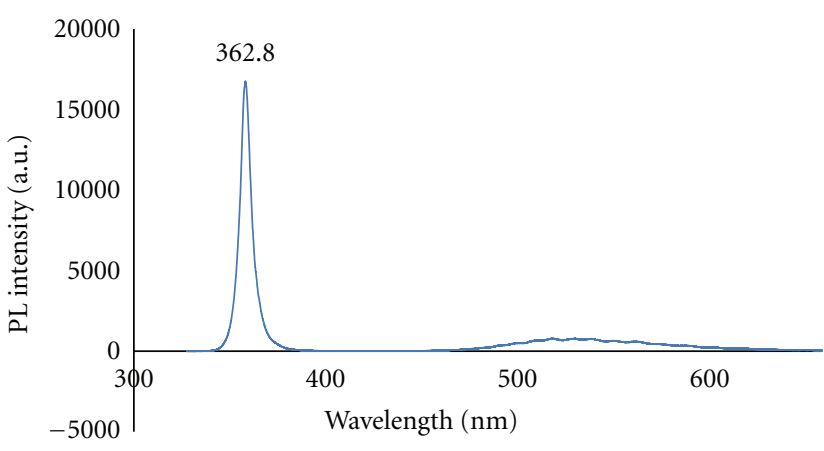

(d)

Figure 4: PL spectrum of the (a) as-grown PZnO, (b) GaN NWs on PZnO, (c) as-grown PGaN, and (d) GaN NWs on the PGaN substrate at room temperature $(300 \mathrm{~K})$.
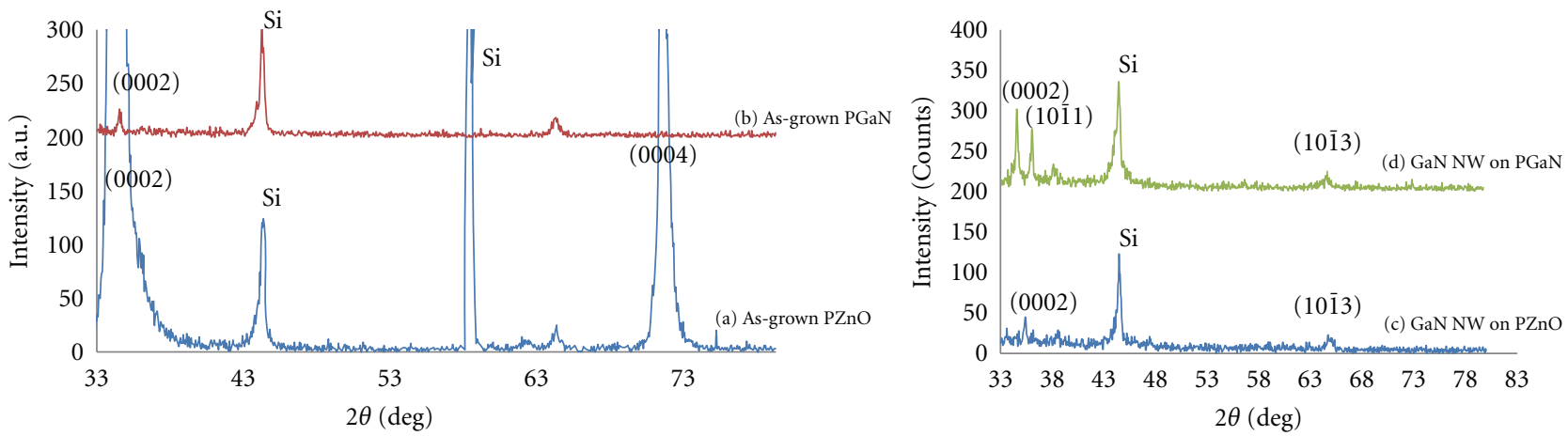

Figure 5: X-ray diffraction of the as-grown (a) PZnO and (b) PGaN wafers and X-ray diffraction of the GaN NWs (c) on PZnO substrate and (d) on PGaN substrate.

intensity (0004). This XRD spectrum mainly shows the $\mathrm{ZnO}$ porous (0002) diffraction peak at $2 \theta=34.3^{\circ}$, whereas Figure 5(b) shows a (0002) higher diffraction peak at $2 \theta=$ $34.5^{\circ}$ for $\mathrm{PGaN}$.

Figure 5(c) shows a typical HR-XRD pattern of the GaN NWs deposited on the PZnO and confirms the formation of single-crystal GaN NWs. The XRD spectrum mainly shows the GaN NWs (0002) diffraction peak at $2 \theta=35^{\circ}$, with full width at half maximum (FWHM) intensity of $0.246^{\circ}$ of the hexagonal wurtzite-type structure.

Figure 5(d) shows another XRD pattern of the GaN NWs deposited on the PGaN. Three peaks are attributed to the GaN NWs: higher (0002), lower (1011), and the lowest
(1013). The XRD spectrum mainly shows the GaN NWs' (0002) diffraction peak at $2 \theta=35^{\circ}$, with FWHM intensity of $0.1968^{\circ}$.

No diffraction peaks from $\mathrm{Ga}_{2} \mathrm{O}_{3}$ or other crystalline impurities could be detected in the samples, which indicate that the samples are of high purity. More GaN NW peaks can be observed on the PGaN substrate than on the PZnO substrate. This indicates the higher quality of the NWs on the PGaN. PGaN has no mismatch with the GaN NWs, making NW formation more pliant than those on PZnO (lattice mismatch between $\mathrm{GaN}$ and $\mathrm{ZnO}$ is $1.8 \%$ ) [18]. Therefore, the XRD spectrum of the NWs on PGaN shows greater number of peaks with higher intensity. 
Considering the SEM images of these NWs, the ratio of the length of the NW $(>10 \mu \mathrm{m})$ to its width $(\sim 40 \mathrm{~nm})$ shows that the growth rate in the [0001] direction is nearly 250 times faster than that of all other directions [19].

It can be suggested that, under the Ar gas flow $(1.5 \mathrm{sccm})$, the $\mathrm{GaN}$ vapor is supersaturated and has a rather small average mean free path. Whenever small crystalline nuclei of GaN NWs are formed, GaN molecules prefer to very quickly self-assemble in the (0001) plane. This kind of preferred one-dimensional growth suggests that the growth pattern corresponds to the fastest way of reducing the system free energy under present nonequilibrium reaction conditions, which means that Ga-N dimer has the most stable structure in the (0001) surface.

If the growth process was conducted in vacuum, the peak intensity would be much better than those obtained in this experiment [20]. The results from the XRD spectra analysis reinforce the conclusions derived from $\mathrm{PL}$ and morphological studies and confirm that the formation of GaN NWs on PGaN is better than that of GaN NWs on $\mathrm{PZnO}$.

\section{Conclusions}

High-density GaN NWs were synthesized on porous $\mathrm{PZnO}$ and PGaN substrates by thermal evaporation method GaN using commercial powder. Thermal evaporation technique is a promising and inexpensive technology for producing GaN NWs, of which the size and distribution can be controlled by changing the growth conditions [4], the size and the porosity of the substrate, duration of growth, and gas flow.

SEM and TEM images show that the diameters of the GaN NWs are in a good range of NWs. The smaller or bigger value of the NW diameter in the case of in-pore growth likely results from the deposition of $\mathrm{GaN}$ on the pore walls at temperatures above $1000^{\circ} \mathrm{C}$ [21]. The morphology of the synthesized NWs reveals that porous substrates play important roles in the fabrication of $\mathrm{GaN}$ nanostructures [22]. The quality of grown GaN NWs for different substrates is highly dependent on the lattice mismatch between the NWs and their substrates.

Results indicate that NWs grown on PGaN have better quality and higher density compared with NWs on PZnO. Structural and optical characterizations by EDX, XRD, and PL show that the growth of the NWs was a function of the semiconductor materials of the porous substrate.

The XRD and EDX spectra show that the NWs are of single-crystal quality with hexagonal wurtzite GaN structure and have preferential [0001] growth direction. PL spectra of the GaN NWs at room temperature show broad and highintensity peak emissions, which indicate that the NWs are of relatively good crystalline and optical quality.

\section{Acknowledgment}

The authors gratefully acknowledge the financial support from the Postgraduate Research Incentive Grant (1001/ PFIZIK/844014) of the Universiti Sains Malaysia.

\section{References}

[1] O. Ambacher, "Growth and applications of group III-nitrides," Journal of Physics D, vol. 31, no. 20, pp. 2653-2710, 1998.

[2] J. Zhang, X. S. Peng, X. F. Wang, Y. W. Wang, and L. D. Zhang, "Micro-Raman investigation of GaN nanowires prepared by direct reaction Ga with NH3," Chemical Physics Letters, vol. 345, no. 5-6, pp. 372-376, 2001.

[3] C.-C. Chen and C.-C. Yeh, "Large-scale catalytic synthesis of crystalline gallium nitride nanowires," Advanced Materials, vol. 12, no. 10, pp. 738-741, 2000.

[4] S. M. Zhou, Y. S. Feng, and L. D. Zhang, "A physical evaporation synthetic route to large-scale GaN nanowires and their dielectric properties," Chemical Physics Letters, vol. 369, no. 5-6, pp. 610-614, 2003.

[5] V. Consonni, M. Knelangen, L. Geelhaar, A. Trampert, and H. Riechert, "Nucleation mechanisms of epitaxial GaN nanowires: origin of their self-induced formation and initial radius," Physical Review B, vol. 81, no. 8, Article ID 085310, 2010.

[6] J. B. Halpern, A. Bello, J. Gilcrease, G. L. Harris, and M. He, "Biphasic GaN nanowires: growth mechanism and properties," Microelectronics Journal, vol. 40, no. 2, pp. 316318, 2009.

[7] X. Duan and C. M. Lieber, "Laser-assisted catalytic growth of single crystal GaN nanowires," Journal of the American Chemical Society, vol. 122, no. 1, pp. 188-189, 2000.

[8] Z. Dong, C. Xue, H. Zhuang et al., "Synthesis of three kinds of $\mathrm{GaN}$ nanowires through $\mathrm{Ga}_{2} \mathrm{O}_{3}$ films' reaction with ammonia," Physica E, vol. 27, no. 1-2, pp. 32-37, 2005.

[9] Y. J. Li, Z. Y. Qiao, and X. L. Chen, "Morphologies of GaN onedimensional materials," Appl Physics A, vol. 71, p. 587, 2000.

[10] M. E. Messing, K. Hillerich, J. Johansson, K. Deppert, and K. A. Dick, "The use of gold for fabrication of nanowire structures," Gold Bulletin, vol. 42, pp. 172-181, 2009.

[11] H. Y. Peng, X. T. Zhou, N. Wang et al., "Bulk-quantity GaN nanowires synthesized from hot filament chemical vapor deposition," Chemical Physics Letters, vol. 327, no. 5-6, pp. 263-270, 2000.

[12] G. S. Cheng, L. D. Zhang, Y. Zhu et al., "Large-scale synthesis of single crystalline gallium nitride nanowires," Applied Physics Letters, vol. 75, no. 16, pp. 2455-2457, 1999.

[13] W. Liu, S. Xing, J. Lian et al., "Microstructure investigation of $\mathrm{Ba}_{x} \mathrm{Sr}_{1-x} \mathrm{TiO}_{3}$ thin film grown on porous silicon substrate," Materials Science in Semiconductor Processing, vol. 7, no. 4-6, pp. 253-258, 2004.

[14] 2007, http://www.hyperscripts.com/semi_en/kap_1/backbone/ r1.html.

[15] B. S. Xu, L. Y. Zhai, J. Liang, S. F. Ma, H. S. Jia, and X. G. Liu, "Synthesis and characterization of high purity GaN nanowires," Journal of Crystal Growth, vol. 291, no. 1, pp. 3439, 2006.

[16] A. Ramizy, Z. Hassan, and K. Omar, "The performance of silicon solar cell with different texturing processes," Sensors and Actuators B, vol. 155, pp. 699-708, 2011.

[17] P. Perlin, C. Jauberthie-Carillon, J. P. Itie, A. San Miguel, I. Grzegory, and A. Polian, "Raman scattering and x-rayabsorption spectroscopy in gallium nitride under high pressure," Physical Review B, vol. 45, no. 1, pp. 83-89, 1992.

[18] T. Suzuki, H. J. Ko, A. Setiawan et al., "Polarity control of GaN epilayers grown on $\mathrm{ZnO}$ templates," Materials Science in Semiconductor Processing, vol. 6, no. 5-6, pp. 519-521, 2003.

[19] L. -B. Zhao, T. -J. Yu, J. -J. Wu, Z. -J. Yang, and G. -Y. Zhang, "Effects of V/III ratio on species diffusion anisotropy in the 
MOCVD growth of non-polar $\alpha$-plane GaN films," Chinese Physics B, vol. 19, no. 1, Article ID 018101, 2010.

[20] Shabnam, C. R. Kant, and P. Arun, "Controlling the photoluminescence of $\mathrm{ZnO}: \mathrm{Si}$ nano-composite films by heattreatment," Materials Research Bulletin, vol. 45, no. 10, pp. 1368-1374, 2010.

[21] D. Buttard, T. David, P. Gentile, F. Dhalluin, and T. Baron, "High-density guided growth of silicon nanowires in nanoporous alumina on $\mathrm{Si}(100)$ substrate: estimation of activation energy," Physica Status Solidi, vol. 3, no. 1, pp. 1921, 2009.

[22] L. Shekari, H. Abu Hassan, A. Ramizy, K. Omar, and Z. Hassan, "Growth and characterization of Wurtzite GaN NWs based-on Porous silicon substrate," in Proceedings of the International Conference of ICAMN, Malaysia, 2010. 

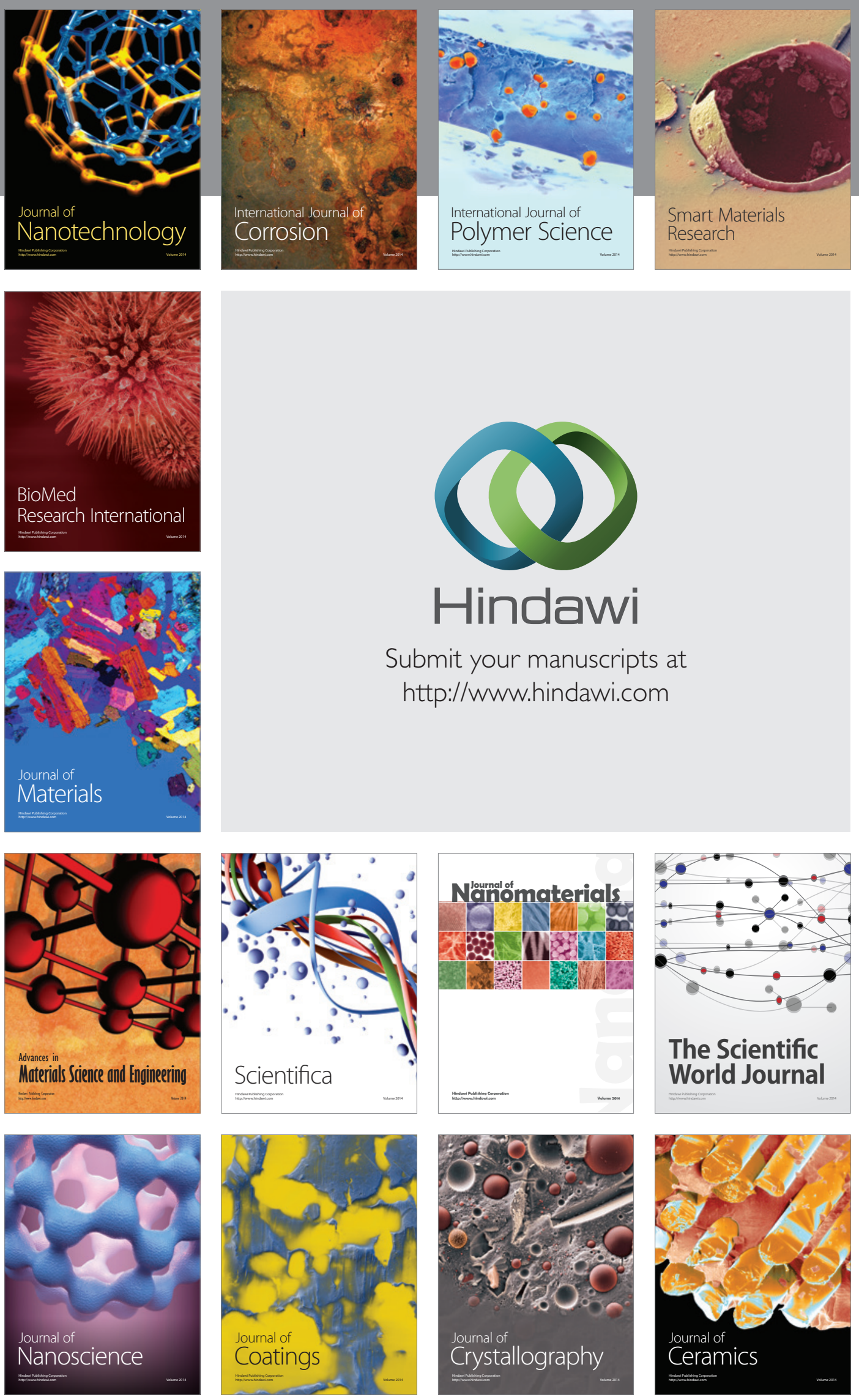

The Scientific World Journal

Submit your manuscripts at

http://www.hindawi.com

\section{World Journal}

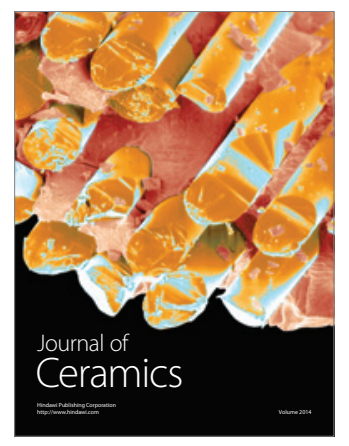

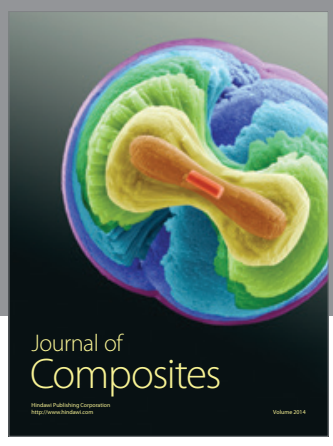
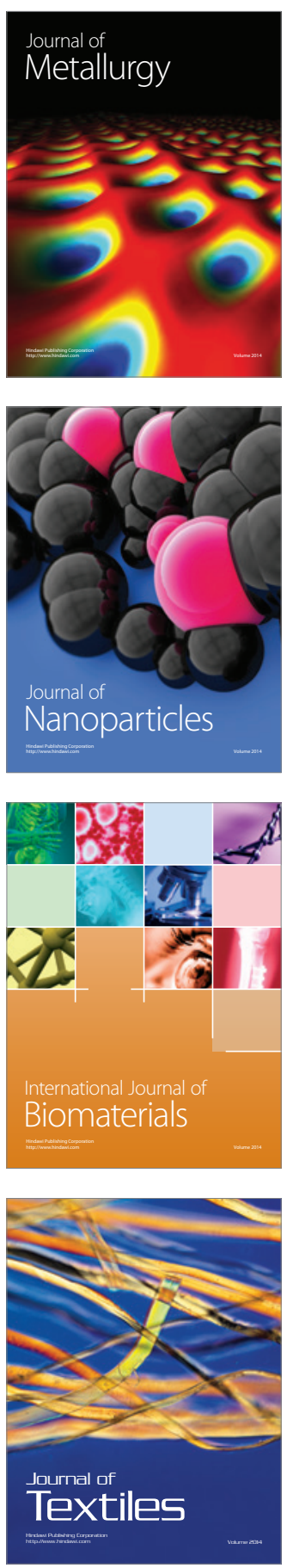